OPEN ACCESS

Edited by:

Daniel Puyol,

King Juan Carlos University, Spain

Reviewed by:

Christopher L. Hemme,

University of Rhode Island, USA

Xiaoke Hu,

Yantai Institute of Coastal Zone Research (CAS), China

${ }^{*}$ Correspondence:

Korneel Rabaey

korneel.rabaey@ugent.be

Specialty section:

This article was submitted to Microbiotechnology, Ecotoxicology

and Bioremediation,

a section of the journal Frontiers in Bioengineering and

Biotechnology

Received: 29 June 2016 Accepted: 02 February 2017 Published: 20 February 2017

Citation:

Andersen SJ, De Groof V, Khor WC,

Roume H, Props R, Coma M and Rabaey K (2017) A Clostridium Group

IV Species Dominates and

Suppresses a Mixed Culture

Fermentation by Tolerance to Medium Chain Fatty Acids Products.

Front. Bioeng. Biotechnol. 5:8. doi: 10.3389/fbioe.2017.00008

\section{A Clostridium Group IV Species Dominates and Suppresses a Mixed Culture Fermentation by Tolerance to Medium Chain Fatty Acids Products}

\author{
Stephen J. Andersen', Vicky De Groof ${ }^{1,2}$, Way Cern Khor ${ }^{1}$, Hugo Roume', Ruben Props ${ }^{1}$, \\ Marta Coma ${ }^{1,2}$ and Korneel Rabaey ${ }^{1 *}$ \\ ${ }^{1}$ Center for Microbial Ecology and Technology (CMET), Ghent University, Gent, Belgium, ${ }^{2}$ Center for Sustainable Chemical \\ Technologies, University of Bath, Bath, UK
}

A microbial community is engaged in a complex economy of cooperation and competition for carbon and energy. In engineered systems such as anaerobic digestion and fermentation, these relationships are exploited for conversion of a broad range of substrates into products, such as biogas, ethanol, and carboxylic acids. Medium chain fatty acids (MCFAs), for example, hexanoic acid, are valuable, energy dense microbial fermentation products, however, MCFA tend to exhibit microbial toxicity to a broad range of microorganisms at low concentrations. Here, we operated continuous mixed population MCFA fermentations on biorefinery thin stillage to investigate the community response associated with the production and toxicity of MCFA. In this study, an uncultured species from the Clostridium group IV (related to Clostridium sp. BS-1) became enriched in two independent reactors that produced hexanoic acid (up to $8.1 \mathrm{~g} \mathrm{~L}^{-1}$ ), octanoic acid (up to $3.2 \mathrm{~g} \mathrm{~L}^{-1}$ ), and trace concentrations of decanoic acid. Decanoic acid is reported here for the first time as a possible product of a Clostridium group IV species. Other significant species in the community, Lactobacillus spp. and Acetobacterium sp., generate intermediates in MCFA production, and their collapse in relative abundance resulted in an overall production decrease. A strong correlation was present between the community composition and both the hexanoic acid concentration $(p=0.026)$ and total volatile fatty acid concentration $(p=0.003)$. MCFA suppressed species related to Clostridium sp. CPB-6 and Lactobacillus spp. to a greater extent than others. The proportion of the species related to Clostridium sp. BS-1 over Clostridium sp. CPB-6 had a strong correlation with the concentration of octanoic acid $(p=0.003)$. The dominance of this species and the increase in MCFA resulted in an overall toxic effect on the mixed community, most significantly on the Lactobacillus spp., which resulted in a decrease in total hexanoic acid concentration to $32 \pm 2 \%$ below the steady-state average. As opposed to the current view of MCFA toxicity broadly leading to production collapse, this study demonstrates that varied tolerance to MCFA within the community can lead to the dominance of some species and the suppression of others, which can result in a decreased productivity of the fermentation.

Keywords: carboxylic acids, Clostridium, fermentation, MCFA, microbial community, toxicity 


\section{INTRODUCTION}

The conversion of a complex substrate in anaerobic digestion and fermentation relies on the competition for carbon and energy within a mixed microbial community to drive the conversion of diverse polymeric biomass to simpler end products, such as methane, acetic acid, and other volatile fatty acids (VFAs). The environmental conditions in such systems are engineered $(\mathrm{pH}$, temperature, organic loading, and wash out rate) in an attempt to optimize performance throughout this collaborative biological economy. Conditions cannot be ideal for each player, and some applied or inherent conditions can result in favorable (or unfavorable) conditions for some species to the detriment (or benefit) of others, thus impacting the role they play in this process. Managing this principle is at the center of engineering a mixed microbial biotechnology (Angenent et al., 2016; Lindemann et al., 2016).

VFAs are bulk chemicals that can be produced in a biorefinery context from organic waste streams. This can include short chain fatty acids such as acetic and propionic acid, common biological intermediates such as lactic acid and succinic acid, and medium chain fatty acids (MCFAs) such as hexanoic and octanoic acid. Chain elongation of short chain VFA occurs through reverse $\beta$-oxidation, in which some species can gain energy by increasing the chain length of VFA with reductive substrates such as ethanol and lactic acid (Seerdorf et al., 2007; Spirito et al., 2014; Angenent et al., 2016). Hexanoic acid has come in focus as a target of bioproduction as it can be used in applications as diverse as antimicrobial animal feed additives and jet fuel precursors. Bioproduction has recently been demonstrated at the laboratory scale at industrially interesting rates (Grootscholten et al., 2013; Ge et al., 2015). Sustainable MCFA production technologies are yet to have reached an industrial scale, but research is on-going and scale-up is underway. Research toward the production of MCFA generally focuses on characterizing the productive species and designing a system based around its preferred conditions and substrates. A body of work exists on Clostridium kluyveri, Megasphaera elsdenii, and other species (Seerdorf et al., 2007; Jeon et al., 2010; Choi et al., 2013; Weimer and Moen, 2013; Ge et al., 2015; Angenent et al., 2016; Kucek et al., 2016), and recently a new genus Caproiciproducens was proposed to house the isolate Clostridum sp. BS-1, under the proposed name of Caproiciproducens galactitolivorans (Kim et al., 2015). The Clostridium group IV has been shown to generate hexanoic acid from lactic acid (Xu et al., 2015).

MCFA have a $\mathrm{pH}$-dependent microbial toxicity that can disrupt membrane integrity at around $40 \mathrm{mM}\left(4.6 \mathrm{~g} \mathrm{~L}^{-1}\right)$ for hexanoic acid $\left(\mathrm{pK}_{\mathrm{a}} 4.85\right)$ and $20 \mathrm{mM}\left(2.9 \mathrm{~g} \mathrm{~L}^{-1}\right)$ for octanoic acid $\left(\mathrm{pK}_{\mathrm{a}} 4.89\right)$, as characterized in Escherichia coli at $\mathrm{pH} 4.3$, with no toxicity demonstrated at pH 7 (Yang et al., 2010; Royce et al., 2013). MCFA fermentation is often performed under acidic conditions to suppress methanogen activity. Stable, continuous operation of a reactor in the presence of MCFA under acidic conditions requires this toxicity to be managed by attentive organic loading, $\mathrm{pH}$ compromise and hydraulic residence time, or extraction by a robust in situ recovery technology that can work effectively at low concentrations to protect the microbial community. Extraction efficiency and unit operation cost are inherently linked to the concentration of the target compound, and as such limited extraction efficiency and high recovery cost are recognized as major practical and economic hurdles in bringing sustainable MCFA production to an industrial reality (Agler et al., 2011; López-Garzón and Straathof, 2014; Andersen et al., 2015; Xu et al., 2015; Angenent et al., 2016).

The supporting microbial community in an MCFA fermentation from a complex substrate is core to the carbon economy. A single-stage production from waste requires microorganisms to perform hydrolysis, acidogenesis, and as necessary, other fermentations (e.g., toward ethanol and lactic acid) (Agler et al., 2011, 2012; Angenent et al., 2016), while also contributing some critical coproduction of gases (e.g., hydrogen gas and carbon dioxide gas). The supporting community in anaerobic digestion includes the Bacteroidetes and Firmicutes phyla, which enrich when switching from biogas to VFA production (De Vrieze et al., 2015). Bacteroidetes have been related to parallel reactions when Clostridium sp. was enriched for MCFA production (Coma et al., 2016). In mixed microbial chain elongation to MCFA, Lactobacillus spp. have also been found in communities alongside Megasphaerea sp. (Andersen et al., 2015) and Clostridium group IV (Zhu et al., 2015), suggesting a lactic acid chain elongation route. Ethanol is often added in studies for mixed culture chain elongation but can also be generated by the supporting community from sugars or acetic acid and carbon dioxide (Spirito et al., 2014). It is arguably somewhat reductive to refer to producers of critical intermediates as a "supporting" community, particularly if the complete utilization of a substrate is critical to the production economics. Within the carbon and energy economy of the reactor microbiome the non-elongating, supporting community is process critical and therefore important to study, understand, and engineer.

Toxicity in an MCFA fermentation, particularly in mixed cultures, is generalized as the broad suppression of microorganisms, leading to the collapse of production. MCFA toxicity is only observed incidentally and, to our knowledge, has not been studied with a mixed culture. This study explores community tolerance and toxicity to MCFA, and by extension the dominance of some MCFA tolerant species and the suppression of others that succumb to the toxicity. In this work, we therefore aimed to describe the microbial relationships in a mixed community MCFA fermentation and specifically investigated how these toxic products impact the community and the process. We describe the operation of two identical reactors fed with biorefinery beer and stillage, an organics-rich stream from the bottoms of a bioethanol distillation column and track changes in the substrate consumption, VFA concentration, and bacterial community composition according to relative abundance.

\section{MATERIALS AND METHODS}

\section{Bioreactor Operation}

Fermentation of beer and stillage from a wheat bioethanol process (supplied by Tereos Starch \& Sweeteners, Aalst, Belgium) was performed in two identical DOLLY twin Bioreactors (Belach 
Bioteknik AB, Sweden). The working volume was fixed at $5 \mathrm{~L}$ and fed at daily intervals from a source stored at $4^{\circ} \mathrm{C}$ and $\mathrm{pH} 3.5 \pm 0.1$. The reactors were maintained at a hydraulic residence time of 7.5 days and $\mathrm{pH} 5.5$, controlled by dosing $5 \mathrm{M} \mathrm{NaOH}$. At start-up, reactors were filled with $4.5 \mathrm{~L}$ adapted DSMZ medium 52 and $0.5 \mathrm{~L}$ of an enriched, in-house inoculum for MCFA production. After $24 \mathrm{~h}, 1.75 \mathrm{~L}$ of medium were replaced with a $5: 2$ beer to stillage ratio mixture to target $10 \mathrm{~g} \mathrm{~L}^{-1}$ ethanol in the total reactor volume, followed by regular HRT feeding at approximately $14 \%$ beer to target an ethanol concentration of $6 \mathrm{~g} \mathrm{~L}^{-1}$. The feed had a measured $57 \pm 9 \mathrm{~g} \mathrm{COD}_{\text {Soluble }} \mathrm{L}^{-1}$ and $101 \pm 18 \mathrm{~g} \mathrm{COD}_{\text {Total }} \mathrm{L}^{-1}$. The concentration of soluble substrates measured included sugars at $9.0 \pm 0.4 \mathrm{~g} \mathrm{~L}^{-1}$ of xylose, $4.9 \pm 0.2 \mathrm{~g} \mathrm{~L}^{-1}$ arabinose and $2.2 \pm 0.1 \mathrm{~g} \mathrm{~L}^{-1}$ glucose $(n=4)$ and $6.0 \pm 2.4 \mathrm{~g} \mathrm{~L}^{-1}$ ethanol, $3.8 \pm 1.2 \mathrm{~g} \mathrm{~L}^{-1}$ glycerol, $1.6 \pm 1.3 \mathrm{~g} \mathrm{~L}^{-1}$ lactic acid, and $0.4 \pm 0.2 \mathrm{~g} \mathrm{~L}^{-1}$ acetic acid, at $\mathrm{pH}$ $3.5 \pm 0.1$ and $6.0 \pm 0.9 \mathrm{~ms} \mathrm{~cm}^{-1}(n=18)$.

\section{Sample Analysis}

Reactors were sampled three times per week and analyzed for C2 to C8 fatty acids (including isoforms C4 to C6) by gas chromatography (GC-2014, Shimadzu ${ }^{\circledR}$, The Netherlands), with a DB-FFAP 123-3232 column $(30 \mathrm{~m} \times 0.32 \mathrm{~mm} \times 0.25 \mu \mathrm{m}$; Agilent, Belgium) and a flame ionization detector (FID). Liquid samples were conditioned with $2 \mathrm{~mL}$ sulfuric acid, $200 \mathrm{mg}$ sodium chloride, and 2-methyl hexanoic acid as an internal standard for quantification before further extraction with diethyl ether (1:1 volume sample:ether). The sample $(1 \mu \mathrm{L})$ was injected at $250^{\circ} \mathrm{C}$ with a split ratio of 50 and a purge flow of $3 \mathrm{~mL} \mathrm{~min}{ }^{-1}$. The oven temperature increased by $10^{\circ} \mathrm{C} \mathrm{min}{ }^{-1}$ from 110 to $250^{\circ} \mathrm{C}$ where it was maintained for $5 \mathrm{~min}$. The FID had a temperature of $300^{\circ} \mathrm{C}$. Nitrogen carrier gas was maintained at a flow rate of $2.49 \mathrm{~mL} \mathrm{~min}{ }^{-1}$. "Total VFA" reported in this study is the quantitative sum of all detected linear, unsaturated carboxylic acids, including MCFA. Decanoic acid (C10) was analyzed by the same method and detected at up to $232 \mathrm{ppm}$, but interference was observed from unidentified compounds already present in the feed, likely to be lipids and lipid fragments. For a comparative analysis of $\mathrm{C} 10$, peaks were normalized between 0 and 1 , with the average integration of the $\mathrm{C} 10$ peak measured in the feed set at 0 $\left(\sigma=7 \times 10^{-4}, n=4\right)$, and the maximum peak set at 1 . Normalized $\mathrm{C} 10$ integrated peaks were clustered into two discrete groupings. Those clustered close to zero are considered a negative detection of the C10 MCFA product, with an average of $-0.03 \pm 0.2(n=6)$, and those outside with an average of $0.60 \pm 0.23(n=6)$ considered a positive detection. The separate clusters were compared for distinctness by $k$-means clustering and compared against C8 concentration, Clostridium sp. BS- $1_{\text {sec }}$ relative abundance, and the ratio of the relative abundance of Clostridium sp. BS- $1_{\text {sec }}$ to Clostridium sp. CPB-6.

The concentrations of lactic acid, formic acid, glycerol, 1,3-propanediol, 1,2-propanediol, methanol, ethanol, propanol, and butanol were analyzed with a 930 Compact IC Flex (Metrohm, Switzerland) ion chromatography system with inline bicarbonate removal (MCS), equipped with an organic acids column (Metrosep 250/7.8; Metrohm) and a guard column cartridge (Metrosep Dual 4/4.6; Metrohm) with a 850 IC conductivity detector. Oven temperature was set at $35^{\circ} \mathrm{C}$. A $1 \mathrm{mM} \mathrm{H}_{2} \mathrm{SO}_{4}$ solution was used as eluent at a flow rate of $0.5 \mathrm{~mL} \mathrm{~min}^{-1}$. The concentration of the lowest standard, determining lower limit of quantification, was $1 \mathrm{mg} \mathrm{L} \mathrm{L}^{-1}$. The concentrations of glycerol, 1,3-propanediol, 1,2-propanediol, ethanol and 2-propanol were analyzed with a 930 Compact IC Flex (Metrohm) ion chromatography system, equipped with an alcohols column (Metrosep Carb 2 250/4.0; Metrohm) and a guard column cartrige (Metrosep Trap 1 100/4.0; Metrohm) with an IC amperometric detector. Oven temperature was set at $35^{\circ} \mathrm{C}$. A $20 \mathrm{mM} \mathrm{NaOH}$ was used as eluent

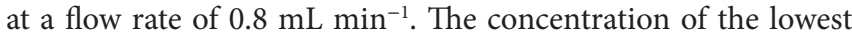
standard used, therefore determining lower limit of quantification, was $0.5 \mathrm{mg} \mathrm{L}^{-1}$ for glycerol, $1 \mathrm{mg} \mathrm{L}^{-1}$ for 1,2-propanediol and 2-propanol, and $5 \mathrm{mg} \mathrm{L}^{-1}$ for 1,3-propanediol and ethanol. The gas-phase composition was analyzed with a Compact GC (Global Analyser Solutions, Breda, The Netherlands), equipped with a Molsieve 5A precolumn and Porabond column $\left(\mathrm{CH}_{4}, \mathrm{O}_{2}\right.$, $\mathrm{H}_{2}$, and $\left.\mathrm{N}_{2}\right)$ and a Rt-Q-bond precolumn and column $\left(\mathrm{CO}_{2}, \mathrm{~N}_{2} \mathrm{O}\right.$, and $\mathrm{H}_{2} \mathrm{~S}$ ). Gases in the headspace were determined by a thermal conductivity detector. $\mathrm{COD}_{\text {Total }}$ was determined according to Standard Methods (APHA, 2005) using the dichromate oxida-

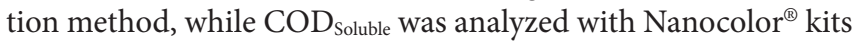
(Macherey-Nagel, Germany) after sample filtration at $0.45 \mu \mathrm{m}$. Sugars were analyzed by the NREL procedure according to Sluiter et al. (2006), measured with high-performance liquid chromatography (Agilent Varian ProStar 220 SDM, USA; $5 \mathrm{mM} \mathrm{H}_{2} \mathrm{SO}_{4}$ mobile phase, $0.6 \mathrm{~mL} \mathrm{~min}^{-1}$ and $60^{\circ} \mathrm{C}$ column temperature with a refractive index detector and Rezex H+ column; Aminex).

\section{Community Analysis}

DNA samples for community analysis of each reactor broth and feed were collected on day 0 (inoculum), days $9,22,34,41,48$, 55 , and 64 and were centrifuged in $2 \mathrm{~mL}$ sterile Micrewtubes ${ }^{\circledR}$ (Simport, Canada). The supernatant was removed, and the samples were stored at $-21^{\circ} \mathrm{C}$. Following DNA extraction using PowerSoil DNA kit (MoBio), 16S rRNA sequences were amplified and sequenced using Illumina sequencing technology as described by Andersen et al. (2015). Bioinformatic analysis of sequences was executed using the Mothur pipeline as described by Andersen et al. (2015). Three samples were chosen for Sanger sequencing of $16 \mathrm{~S}$ rRNA by LGC genomics (Berlin, Germany) to identify enriched cultures on the $16 \mathrm{~S}$ level. These samples were chosen due to their high relative abundance of the uncultured species Clostridium sp. BS- $1_{\text {sec }}$, with a relative abundance of approximately $80 \%$ from days 34 and 41 from Reactor 1 , and day 62 from Reactor 2. Only day 34 from Reactor 1 returned a valid sequence. The alignment considered 974 bp of the phylogenetic gene maker $16 \mathrm{~S}$ rRNA. The alignment file was submitted to a phylogenetic analysis using the Phylogeny.fr customized workflow service1 including alignment curation with Gblocks2 (using default parameters), tree construction with PhyML3 (using default parameters), and visualization by TreeDyn4 (Castresana, 2000; Chevenet et al., 2006; Dereeper et al., 2008; Guindon et al., 2010). Consensus sequences of all OTUs are available in supplementary information, and raw sequence data are available from the European Nucleotide Archive (ENA) under study accession number PRJEB19351. 


\section{Statistics}

Multivariate abundance analysis was used for statistical inference on the relationship between relative species abundances and VFA. All analyses were conducted in R (v3.2.5) with seed 777 using the mvabund package (Wang et al., 2012). Samples were rarefied until the minimum sample depth (9,848 reads) and pruned from operational taxonomic units (OTUs), which had a maximum relative abundance lower than $10 \%$ or were absent in at least $50 \%$ of the samples to specifically focus on the dominant bacterial species. A forward selection-based modeling approach was used. The mean-variance relationship was modeled by a negative binomial distribution, and all models were verified for accordance with the model assumptions as described by Wang et al. (2012). Hypothesis testing was performed using likelihood ratio tests with pit resampling (5,000 runs). The final model consisted of hexanoic acid and total VFA concentration as continuous predictors and the reactor replicate as categorical predictor. Inference on the model parameters of individual species was executed using the adjusted $p$-values, calculated after 5,000 resampling runs to account for family-wise error rates and intervariable correlations. Ordination plots were made using the decorana and rda functions available in the vegan package (Oksanen et al., 2015). Detrended correspondence analysis (decorana) was applied on the rarefied community data to avoid horseshoe artifacts that are frequently associated with time series community data. Principal coordinate analysis was used on the VFA dataset.

\section{RESULTS}

\section{Production of MCFAs and the Collapse of Glycerol Consumption}

Hexanoic acid was consistently produced in both bioreactors at $\mathrm{pH} 5.5$, with a steady-state concentration of $2.0 \pm 1.1 \mathrm{gC} \mathrm{L}^{-1}$ $\left(3.2 \pm 1.8 \mathrm{~g} \mathrm{~L}^{-1}\right)$ in Reactor 1 and $3.2 \pm 0.9 \mathrm{gC} \mathrm{L}^{-1}\left(5.2 \pm 1.5 \mathrm{~g} \mathrm{~L}^{-1}\right)$ in Reactor 2 (Figure 1), including a maximum concentration of $5.0 \mathrm{gC} \mathrm{L}^{-1}\left(8.0 \mathrm{~g} \mathrm{~L}^{-1}\right)$ hexanoic acid on day 22 in Reactor 2. As a proportion of the total VFA on a carbon basis, $54 \pm 10 \%$ of the VFA present was hexanoic, heptanoic, or octanoic acid in Reactor 1 and $45 \pm 10 \%$ in Reactor 2 (all averages are based on the steady-state period of days $15-68, n=13)$. The VFA profile of the identical reactors began to diverge at day 20 , with Reactor 1 progressively increasing to a maximum of $2.1 \mathrm{gC} \mathrm{L}^{-1}$ of octanoic acid by day 39. The total VFA concentration decreased from $7.35 \mathrm{gC} \mathrm{L}^{-1}$ on day 20 to a low of $3.46 \mathrm{gC} \mathrm{L}^{-1}$ by day $46,36 \%$ lower than the average steady-state concentration. In Reactor 1 , this decrease coincided with a change in glycerol consumption. Before day 20, there was an almost complete consumption of glycerol, which shifted to zero net change after day 20, i.e., glycerol detected in the feed was equal to glycerol concentration in the effluent. Reactor 2, operated identically and fed in parallel from the same source, did not show this trend at this time, but rather maintained a total concentration of $7.7 \pm 0.6 \mathrm{gC} \mathrm{L}^{-1}$ for the days 15-57 with a hexanoic acid concentration of $3.5 \pm 0.8 \mathrm{gC} \mathrm{L}^{-1}$, and a heptanoic and octanoic concentration between 0 and $0.2 \mathrm{gC} \mathrm{L}^{-1}$. The octanoic acid concentration began to rise in Reactor 2 after day 60, which coincided with a decline in both hexanoic acid and the total VFA concentration from $7.4 \mathrm{gC} \mathrm{L}^{-1}$ on day 60 to a low of $5.0 \mathrm{gC} \mathrm{L}^{-1}$ by day 68 , $32 \%$ less than the total average steady-state VFA concentration. Decanoic acid was detected in Reactor 1 on days 22 and 41-64 with a relative peak average of $0.50 \pm 0.01(n=6)$, significantly higher than the baseline of the feed at $0 \pm 7 \times 10^{-4}(n=4)$. Decanoic acid was not detected significantly above the baseline in Reactor 2 with a relative peak average of $0.05 \pm 2 \times 10^{-3}$ $(n=6)$, with the exception for day 67. Across all samples, decanoic acid detection occurs only when octanoic acid is detected (Figure 1), generally when the concentration of octanoic acid is above around $1 \mathrm{gC} \mathrm{L}^{-1}$ (Figure 1; Figure $\mathrm{S} 1$ in Supplementary Material).

\section{Community Domination by Tolerance to MCFA}

In both reactors, the community was dominated by Clostridium and Lactobacillus species (Figures 1C,D). Multivariate abundance testing shows that the community composition was significantly associated with the hexanoic acid concentration $(p=0.026)$ and total VFA concentration $(p=0.003)$ for the community of both reactors, with a significantly different community composition between the two identical reactors $(p=0.05)$. These overall differences could be significantly attributed to the Lactobacillus spp. (Otu0001) $(p=0.002)$, which generate lactic acid that the Clostridia species are likely to metabolize (Jeon et al., 2010; Kim et al., 2015; Kucek et al., 2016). The two reactors had a high functional similarity in terms of VFA output (Figure S5A in Supplementary Material) despite a large dissimilarity in community profile throughout the 70 days of operation (Figure S5B in Supplementary Material). The peak of dissimilarity in functionality corresponded to the increase of octanoic acid in Reactor 1, and the functional dissimilarity begins to fall as the concentration of octanoic acid in Reactor 2 begins to rise.

The two reactors were dominated by an uncultured Clostridium species (Otu004) with 95\% similarity to Clostridium sp. BS-1 over 427 bp (Table S2 in Supplementary Material). This OTU correlates with the concentration of hexanoic acid and total VFA (Figure 2), and the relative abundance of this species coincides with periods of high octanoic acid and the detection of decanoic acid in both reactors (Figure 1; Figure S2 in Supplementary Material). This species will be referred to as Clostridium sp. BS- $1_{\text {sec }}$ (i.e., secundum) for brevity, as although it is a relation to Clostridium sp. BS-1, the results do not conclusively identify this strain as such. Clostridium sp. BS-1 was recently identified as a D-galactitol consuming, fermentive microorganism capable of producing acetic acid, butyric acid, and hexanoic acid (Jeon et al., 2010). Another highly abundant OTU was identified as Clostridium sp. CPB-6 (Otu008) with $100 \%$ similarity over $427 \mathrm{bp}$, and this OTU showed a correlation with heptanoic and octanoic acid and high total chain elongation (i.e., the cumulative total concentration of VFA greater than C5 chain length) (Figure 2). As yet, no further information exists on the Clostridium sp. CPB-6 strain, although considering it is 

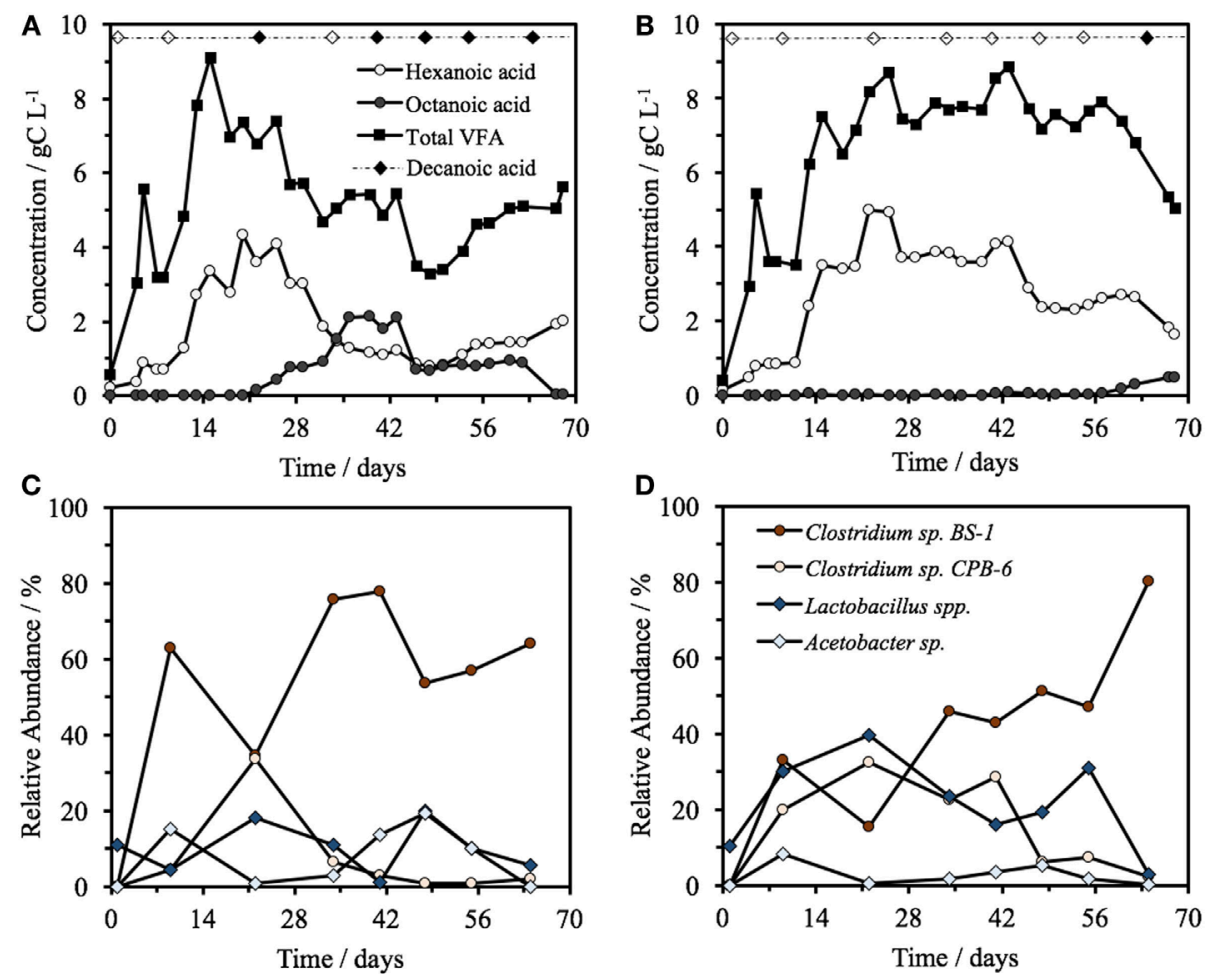

FIGURE 1 | (A,B) Concentration over time of total VFA, hexanoic acid, and octanoic acid in Reactor 1 (A) and Reactor 2 (B) fed from a common source of biorefinery stillage, including the detection of decanoic acid. The filled diamond represents the days at which decanoic acid was detected significantly above the feed baseline, with a blank diamond representing no significant detection. The top four most relatively abundant microorganisms over time in the two identical reactors, Reactor 1 (C) and Reactor 2 (D)

closely related to Clostridium sp. BS-1 and likely a member of the Clostridium group IV (96\% similarity identified in Otu022), this species may also be able to produce MCFA through chain elongation. Clostridium sp. BS- $1_{\text {sec }}$ had the greatest relative abundance in both reactors with the exception of day 20 in Reactor 2. In the most stable period of hexanoic production, Reactor 2 had an average Clostridium sp. BS- $1_{\text {sec }}$ relative abundance between 15 and 46\%, the relative abundance of Clostridium sp. CPB-6 was between 22 and 32\%, and Lactobacillus spp. between 16 and 40\%. Lactobacillus spp. are fermentive microorganisms than can convert a wide variety of substrates into lactic acid, including glycerol (Cantoni and Molnar, 1967). Lactic acid can be used by some microorganisms, including some species in the Clostridium group IV (Zhu et al., 2015) to elongate the VFA chain from acetic acid (C2) through butyric acid (C4) to hexanoic acid and longer. Some Lactobacillus are able to degrade lactic acid into acetic acid, particularly if oxygen is available as the electron acceptor (Quatravaux et al., 2006). Acetobacter sp., present in both reactors, is an obligate aerobe that is able to convert ethanol into acetic acid in the presence of oxygen (Cleenwerck et al., 2002). The reactors were fed from an identical source, an undiluted mixture of biorefinery beer and stillage that was not stored anaerobically, which could account for the abundance and survival of this bacteria. The feed had an average $(n=6)$ relative abundance of $72 \pm 19 \%$ for Lactobacillus spp.,
$22 \pm 19 \%$ for Acetobacter sp., $3 \pm 5 \%$ for Gluconobacter sp., and $3 \pm 1 \%$ for other species.

\section{The Uncultured Species Related to the Clostridium Group IV}

A phylogenetic tree analysis (Figure 3) of the Clostridium species referred to as Clostridium $\mathrm{sp}$. BS- $1_{\mathrm{sec}}$ was generated through long $16 \mathrm{~S}$ rRNA sequencing based on a sample from Reactor 1 on day 34 in which its relative abundance was $76 \%$ (Figure 1). Clostridium sp. BS- $1_{\text {sec }}$ has a $95 \%$ maximum similarity with Clostridium sp. BS-1 according to the NCBI BLAST database, with a close relationship to Clostridium sp. CPB-6. Both of these Clostridia appear to be of the Clostridium group IV, recognized as butyrate producers in the gut microbiome (Lopetuso et al., 2013) and capable of elongation of short chain VFA through lactic acid to hexanoic acid (Zhu et al., 2015).

\section{DISCUSSION}

\section{MCFA Toxicity Decreases Hexanoic Acid Production by Suppressing the Supporting Community}

One must take care when implying function from relative abundance, however, some inferences can be made by correlating the 


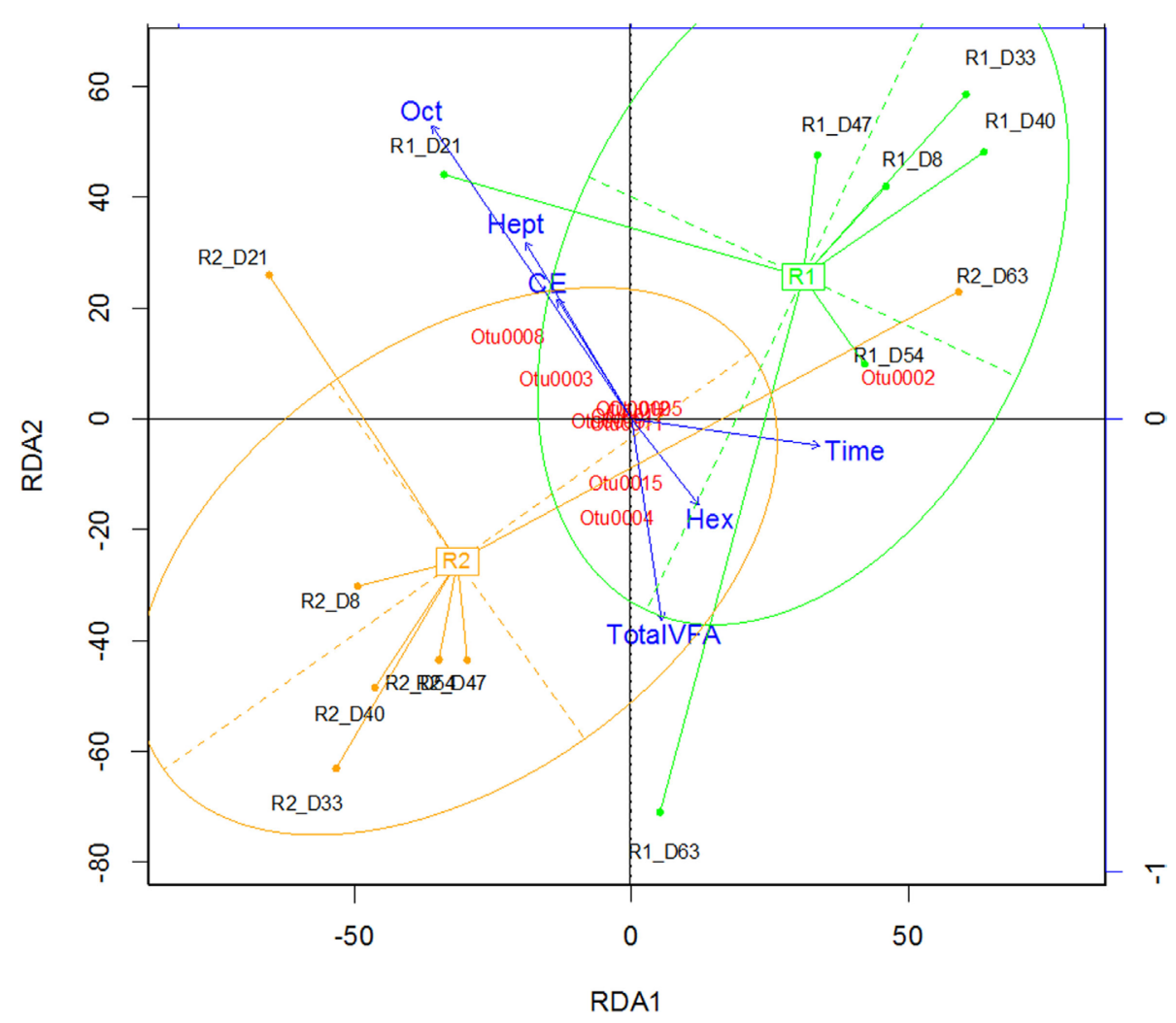

FIGURE 2 | Redundancy analysis demonstrating the alignment of octanoic acid (Oct), heptanoic acid (Hep), and CE (chain elongation, i.e., concentration of volatile fatty acids greater than C5 chain length) in the opposite direction to the total VFA and hexanoic acid (Hex) concentration. Rx_Dy corresponds to Reactor $x$ on day $y$.

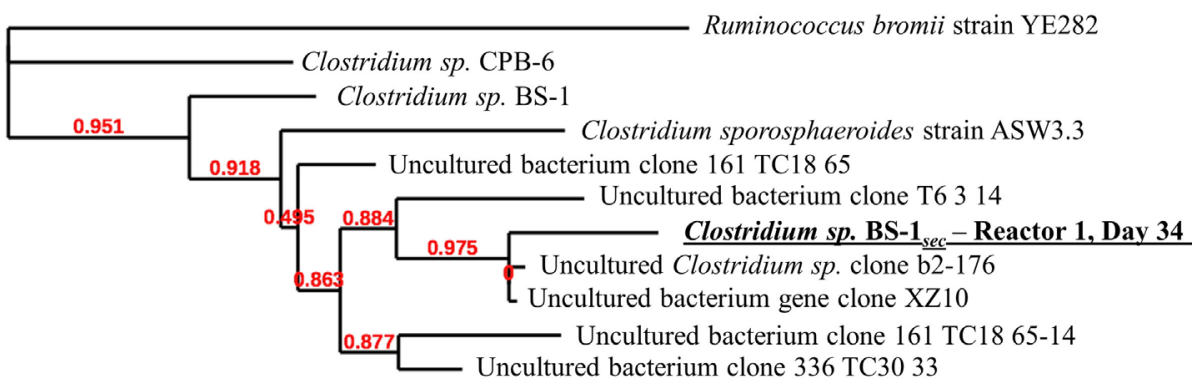

0.1

FIGURE 3 | Phylogenetic tree at species-level resolution showing some genetic heterogeneity among close relatives of the dominant sequence of

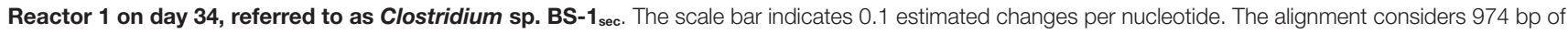
the phylogenetic gene maker16S rRNA. See Table S1 in Supplementary Material for more information.

VFA products with relative abundance, combined with functional knowledge from previous studies. Clostridium sp. BS- $1_{\mathrm{sec}}$ is the only species identified with a high relative abundance that is recognized to produce hexanoic acid, likely through the reverse $\beta$-oxidation pathway. Reverse $\beta$-oxidation can in principal generate octanoic acid and decanoic acid, though decanoic acid has not been previously reported as a product of the reverse $\beta$-oxidation pathway. As a close relative, Clostridium sp. CPB-6 is likely to also produce hexanoic acid, but there appears to be an antagonistic relationship between the two species. There is a significant linear correlation between the concentration of octanoic acid and the ratio of Clostridium sp. BS- $1_{\text {sec }}$ to Clostridium sp. CPB-6 $\left(R^{2}=0.63, p=0.003, n=12\right)$ and a weaker but still significant correlation for the peaks of decanoic acid $\left(R^{2}=0.25\right.$, 
$p=0.006, n=12)$. When both species have similar proportions, the concentration of octanoic acid is low, however, the further Clostridium sp. BS- $1_{\text {sec }}$ exceeds Clostridium sp. CPB-6 in relative abundance, the greater the concentration of octanoic acid. This may be a result of MCFA toxicity decreasing the relative abundance of Clostridium sp. CPB-6 or the capacity of Clostridium sp. BS- $1_{\text {sec }}$ to thrive by generating MCFA through the reverse $\beta$-oxidation pathway. Octanoic acid has been reported as product of C. kluyveri in mixed cultures (Steinbusch et al., 2011; Spirito et al., 2014), however not explicitly confirmed for any species in pure culture studies. Basic $k$-means clustering the samples (Figures S1, S2, and S4 in Supplementary Material) distinctly separates the negative $\mathrm{C} 10$ detection case from the positive case, with the positive $\mathrm{C} 10$ peak clusters cooccurring with high concentrations of $\mathrm{C} 8$, high relative abundance of Clostridium sp. BS $-1_{\mathrm{sec}}$, and a high ratio of Clostridium $\mathrm{sp}$. BS- $1_{\mathrm{sec}}$ to Clostridium sp. CPB-6. This supports the hypothesis that the domination of Clostridium sp. BS- $1_{\text {sec }}$ leads to C8 and C10 MCFA, but here we cannot conclusively state that $\mathrm{C} 10$ is a result of reverse $\beta$-oxidation production, as opposed to lipid fragments from dead cells (such as yeast or Lactobacillus spp.) overlapping the C10 detection.

Clostridium sp. BS- $1_{\text {sec }}$ is clearly tolerant to MCFA as the only species that increases in relative abundance during moments of high hexanoic and octanoic acid concentration, up to a maximum relative abundance of $78 \%$ in Reactor 1 and $80 \%$ in Reactor 2. Some species within the Clostridium group IV have been observed to remain productive up to a hexanoic acid concentration of $23.4 \mathrm{~g} \mathrm{~L}^{-1}$ at $\mathrm{pH}$ 6-6.5, accounting for between 0.5 and $1.5 \mathrm{~g} \mathrm{~L}^{-1}$ of undissociated acid (Zhu et al., 2015). Considering the $\mathrm{pK}_{\mathrm{a}}$ of hexanoic acid, this is in the same vicinity as the upper limit of $0.87 \mathrm{~g} \mathrm{~L}^{-1}$ of protonated hexanoic acid reported by Angenent et al. (2016). Here, with operation at $\mathrm{pH} 5.5$, one expects a protonated hexanoic and octanoic acid proportion of around $18-20 \%$ or an average steady-state concentration of $0.6 \pm 0.3 \mathrm{~g} \mathrm{~L}^{-1}$ protonated hexanoic acid in Reactor 1 and $1.0 \pm 0.3 \mathrm{~g} \mathrm{~L}^{-1}$ in Reactor 2, with the maximum in Reactor 2 reaching $1.46 \mathrm{~g} \mathrm{~L}^{-1}$. The maximum concentration of undissociated octanoic acid across both reactors is approximately $0.2 \mathrm{~g} \mathrm{~L}^{-1}$, and even at these low concentrations, the supporting community seem to be rather sensitive to this acid. In Reactor 1 , Clostridium sp. CPB- 6 reached a relative abundance of $34 \%$ by day 20 and then fell to between 1 and $7 \%$ when the octanoic acid concentration rose in the broth. During this period, the relative abundance of Lactobacillus spp. shifted between 1 and 20\%, with the relative abundance minimum associated with the octanoic acid concentration maximum. In Reactor 2, as Clostridium sp. CPB-6 decreased, Acetobacter sp. increased in relative abundance to between 3 and $20 \%$ from days 20 to 55, before decreasing to less than $1 \%$ by the end of the experiment when octanoic acid was at its greatest concentration in this reactor. The relative abundance of Clostridium sp. BS- $1_{\text {sec }}$ rose to a maximum of $80 \%$ in Reactor 2 at this octanoic acid maximum, with the relative abundance of Clostridium sp. CPB-6 at 2\% and Lactobacillus spp. at $3 \%$. The Lactobacillus spp. Otu0001 was identified as having a statistically significant association with the total VFA concentration $(p=0.0016)$, which indicates that it plays a significant role in carbon conversion. As mentioned, in Reactor 1, there was a neartotal consumption of glycerol until day 25 , followed by a shift to almost zero as the octanoic acid concentration rises. This is an indication that the species responsible for glycerol consumption, likely to be Lactobacillus spp., were suppressed by octanoic acid and other MCFA (illustrated in Figure 4).

\section{The Divergence of Identical Reactors}

The two identical reactors fed from a common source diverged in the community according to relative abundances, suggesting a rather stochastic community response under the highly complex feed and diverse mixed microbial population. The onset of octanoic and decanoic acid products cannot necessarily be linked to a discrete event in the reactor, however, the variability of the community and the abundance of MCFA producers presented conditions suitable for a "runaway reaction"- - a decidedly deterministic toxicity of the supporting community, which drove the Clostridium sp. BS- $1_{\text {sec }}$ to dominance. The two reactors tended toward a low functional dissimilarity, despite the consistently

\section{A Non-inhibited substrate pathway}

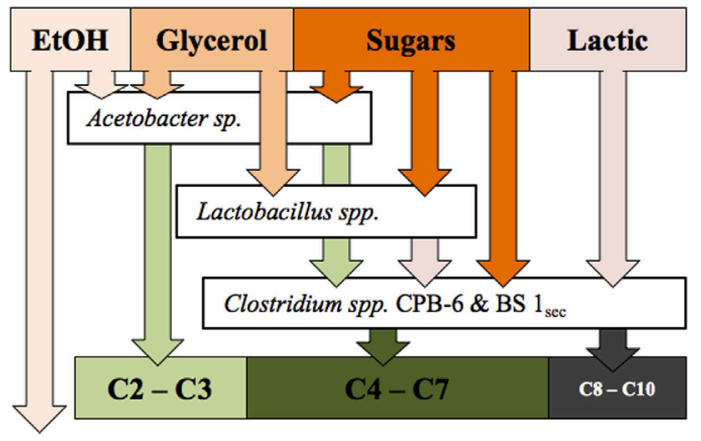

\section{B Impact of MCFA toxicity on substrate pathway}

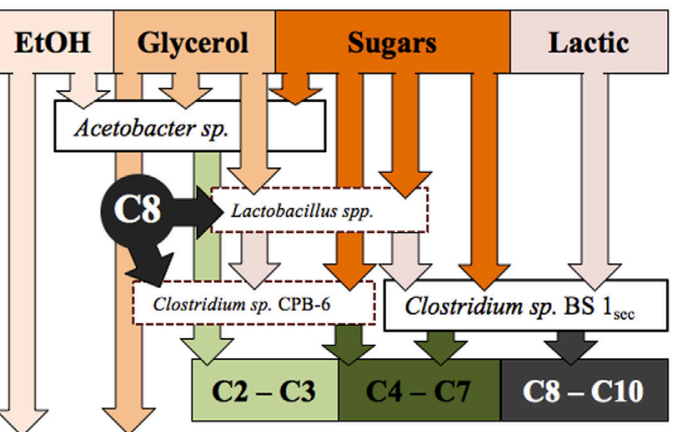

FIGURE 4 | (A) The proposed substrate pathway for the fermentation prior to the onset of MCFA toxicity, as observed days 14-21 in Reactor 1 and days 14-52 in Reactor 2. (B) The hypothetical impact of MCFA toxicity on the proposed substrate pathway, as observed from days 21 to 68 in Reactor 1 . The dashed line borders represent stressed, low relative abundance species. 
high dissimilarity of the community. The ability of the Clostridium group IV species to take advantage of the short chain VFA end products of other organisms through chain elongation gives them this advantage to runaway to dominance and puts the supporting community at risk of the MCFA products.

Analogous systems in anaerobic digestion and other environmental ecologies often show a high degree of stochastic community turnover prior to a significant environmental disturbance (Zhou et al., 2013; Meerburg et al., 2016), while MCFA fermentation in a mixed community has been shown to be shaped non-randomly in a system with an in situ liquid/ liquid extraction system, specifically targeting the hydrophobic MCFA (Agler et al., 2012). The toxicity of MCFA on a mixed community, as shown in this study, has a significant impact on the community with a deterministic downturn in overall production.

\section{What Is the Limit of Reverse $\beta$-Oxidation Chain Elongation?}

Octanoic acid has been previously detected in mixed community MCFA fermentations (Steinbusch et al., 2011; Van Eerten-Jansen et al., 2013; Spirito et al., 2014) but has not been conclusively identified as a product of reverse $\beta$-oxidation. This is partly due to the fact that it is not commonly expected as a likely product and therefore not necessarily included in analysis. Hexanoic acid production has been observed through reverse $\beta$-oxidation at up to $23.4 \mathrm{~g} \mathrm{~L}^{-1}$ (Zhu et al., 2015), which entertains the possibility that some of these microorganisms attempt to exact more energy by sending hexanoic acid through the reverse $\beta$-oxidation again. The fermentation by Zhu et al. (2015) was dominated by Clostridium group IV species engaged in lactic acid elongation, and it is not reported if the broth was tested for other MCFA. While reports of octanoic acid production are rare and exclusive to mixed culture fermentations, reports of decanoic acid as a microbial product are non-existent, to the author's best knowledge. In this study, the cooccurrence of the C10 peaks with a high relative abundance of Clostridium sp. BS- $1_{\text {sec }}$ and high concentrations of octanoic acid adds weight that decanoic acid may be an unreported reverse $\beta$-oxidation product that occurs once a threshold of octanoic acid has been passed, similar to the elongation of acetic acid to butyric, to hexanoic, and so on. The elongating species, in this case likely to be Clostridium sp. BS- $1_{\text {sec }}$ (but perhaps also Clostridium sp. CPB-6), has outcompeted the flanking community to the point at which the available carbon and energy has becomes limited. One counter argument is the collapse of the flanking community, such as Lactobacillus spp., that coincides with the onset of the detection of octanoic acid or with sustained concentrations of hexanoic acid. Cellular fatty acid fragments from the dead cells may fit some of the correlations used here to support the C10 through reverse $\beta$-oxidation hypothesis, such as the increase in relative abundance of Clostridium sp. BS- $1_{\text {sec }}$, which incounter can be viewed as the collapse in relative abundance of all other species. For example, the cellular fatty acid profile of Lactobacillus spp. can contain 2-hexyl-cyclopropanedecanoic acid (lactobacillic acid, $\mathrm{C}_{19} \mathrm{H}_{36} \mathrm{O}_{2}$ ), which contains a decanoic acid chain.

\section{Engineering and Understanding Complex, Mixed Culture MCFA Fermentation}

Environmental factors such as temperature, $\mathrm{pH}$, organic loading, and residence time, and to a certain extent, the mixed community inoculum, are under our control in an engineered system. In this study, all these factors were controlled in two identical reactors, chosen to be most conducive to the production of hexanoic acid, as is common practice in a mixed microbial community anaerobic MCFA fermentation. Though the two reactors had similar functional similarity, the community dissimilarity that arose led to Clostridium sp. BS- $1_{\text {sec }}$ becoming the most relatively abundant microorganism, with octanoic and decanoic acid following and suppressing the activities of the rest of the community to the detriment of overall production. Lactobacillus spp. in particular decreased in relative abundance, which is likely to have resulted in a negative impact on conversion of the stillage into VFA. At the highest concentration of octanoic acid (day 39) in Reactor 1, the total VFA concentration was $59 \%$ of the total maximum VFA concentration (day 15). This phenomenon arose independently in the two identical, separate reactors operated in parallel, with the effect separated temporally by around 35 days. The highest concentration of hexanoic acid occurred in Reactor 2, and coincided with a minimum relative abundance of one of the likely producers of hexanoic acid, Clostridium sp. BS- $1_{\text {sec }}$, during a period where Clostridium sp. BS- $1_{\text {sec }}$, Clostridium sp. CPB-6, and Lactobacillus spp. each had a similar relative abundance in the vicinity of $15-40 \%$. The greatest relative abundances of Clostridium sp. BS- $1_{\text {sec }}$ coincided with a low hexanoic acid concentration and a low total VFA concentration. The system performed at its best-high conversion of substrate and a large proportion of hexanoic acid-when different species coexisted, while the presence of octanoic acid reduced the richness of the microbial community. Rather than optimizing reactor conditions to the benefit of Clostridium sp. BS- $1_{\text {sec }}$, perhaps a better approach is ensuring a diverse biological economy to optimize anaerobic digestion and fermentation, alongside a targeted extraction of the problem compounds, if possible.

It is complicated to parse the origin of octanoic and decanoic acid, the rise in the relative abundance of Clostridium sp. BS- $1_{\mathrm{sec}}$, and the equal and opposite decrease in relative abundance of the other species, but the cooccurrence of Clostridium sp. BS- $1_{\text {sec }}$ with the toxic octanoic acid product highlights the process risk of a winner-takes-all approach and encourages research into the interplay of the complex and competitive microbial community to engineer a stable, productive process that generate sustainable, valuable biochemicals. Toxicity in an MCFA fermentation, particularly in mixed cultures, has heretofore been viewed as the broad suppression of microorganisms leading to the collapse of production. In current literature, MCFA toxicity is only observed incidentally and, to our knowledge, has not been studied with a mixed culture. This study demonstrates varied tolerance to MCFA, and this variation within the community can lead to the dominance of some species while others succumb to the toxicity, which has a significant impact on the productivity of the fermentation. 


\section{AUTHOR CONTRIBUTIONS}

SA designed and executed the experiments, analyzed the results, and wrote the manuscript. VD and WK executed some experiments and analyzed the results. RP and HR performed molecular analysis and designed and executed some statistical analyses. MC and KR contributed in design of experiments and interpretation of the results. All authors contributed to the final manuscript.

\section{ACKNOWLEDGMENTS}

The authors would like to thank Tereos Starch \& Sweeteners, Aalst, Belgium, for generously providing the thin stillage used in this study. The authors warmly thank Emma Hernandez Sanabria and Alberto Scoma for critically reading the manuscript and providing additional valuable insights.

\section{REFERENCES}

Agler, M. T., Spirito, C. M., Usack, J. G., Werner, J. J., and Angenent, L. T. (2012). Chain elongation with reactor microbiomes: upgrading dilute ethanol to medium-chain carboxylic acids. Energ. Environ. Sci. 5, 8189-8192. doi:10.1039/ C2EE22101B

Agler, M. T., Wrenn, B. A., Zinder, S. H., and Angenent, L. T. (2011). Waste to bioproduct conversion with undefined mixed cultures: the carboxylate platform. Trends Biotechnol. 29, 70-78. doi:10.1016/j.tibtech.2010.11.006

Andersen, S. J., Candry, P., Basadre, T., Khor, W. C., Roume, H., Hernandez Sanabria, E., et al. (2015). Electrolytic extraction drives volatile fatty acid chain elongation through lactic acid and replaces chemical $\mathrm{pH}$ control in thin stillage fermentation. Biotechnol. Biofuels 8, 221. doi:10.1186/s13068-015-0396-7

Angenent, L. T., Richter, H., Buckel, W., Spirito, C. M., Steinbusch, K. J. J., Plugge, C. M., et al. (2016). Chain elongation with reactor microbiomes: open-culture biotechnology to produce biochemicals. Environ. Sci. Technol. 50, 2796-2810. doi:10.1021/acs.est.5b04847

APHA. (2005). Standard Methods for the Examination of Water and Wastewater. Washington, DC: American Public Health Association.

Cantoni, C., and Molnar, M. R. (1967). Investigations on the glycerol metabolism of Lactobacilli. J. Appl. Microbiol. 30, 197-205. doi:10.1111/j.1365-2672.1967. tb00289.x

Castresana, J. (2000). Selection of conserved blocks from multiple alignments for their use in phylogenetic analysis. Mol. Biol. Evol. 17, 540-552. doi:10.1093/ oxfordjournals.molbev.a026334

Chevenet, F., Brun, C., Bañuls, A.-L., Jacq, B., and Christen, R. (2006). TreeDyn: towards dynamic graphics and annotations for analyses of trees. BMC Bioinformatics 7:439. doi:10.1186/1471-2105-7-439

Choi, K., Jeon, B. S., Kim, B. C., Oh, M. K., Um, Y., and Sang, B. I. (2013). In situ biphasic extractive fermentation for hexanoic acid production from sucrose by Megasphaera elsdenii NCIMB 702410. Appl. Biochem. Biotechnol. 171, 1094-1107. doi:10.1007/s12010-013-0310-3

Cleenwerck, I., Vandemeulebroecke, D., Janssens, D., and Swings, J. (2002). Re-examination of the genus Acetobacter, with descriptions of Acetobacter cerevisiae sp. nov. and Acetobacter malorum sp. nov. Int. J. Syst. Evol. Microbiol. 52, 1551-1558. doi:10.1099/ijs.0.02064-0

Coma, M., Vilchez-Vargas, R., Roume, H., Jauregui, R., Pieper, D. H., and Rabaey, K. (2016). Product diversity linked to substrate usage in chain elongation by mixed-culture fermentation. Environ. Sci. Technol. 50, 6467-6476. doi:10.1021/ acs.est.5b06021

De Vrieze, J., Saunders, A. M., He, Y., Fang, J., Nielsen, P. H., Verstraete, W., et al. (2015). Ammonia and temperature determine potential clustering in the anaerobic digestion microbiome. Water Res. 75, 312-323. doi:10.1016/ j.watres.2015.02.025

Dereeper, A., Guignon, V., Blanc, G., Audic, S., Buffet, S., Chevenet, F., et al. (2008). Phylogeny. fr: robust phylogenetic analysis for the non-specialist. Nucleic Acids Res. 36, W465-W469. doi:10.1093/nar/gkn180

\section{FUNDING}

SA, MC, and KR are supported by Ghent University Multidisciplinary Research Partnership (MRP) - Biotechnology for a sustainable economy (01 MRA 510W). WK is supported by Het Bijzonder Onderzoeksfonds (BOF, DEF13/AOF/010). KR and HR are supported by European Research Council Starter Grant ELECTROTALK. RP is supported by Ghent University (BOFDOC2015000601) and the Belgian Nuclear Research Centre (SCK.CEN).

\section{SUPPLEMENTARY MATERIAL}

The Supplementary Material for this article can be found online at http://journal.frontiersin.org/article/10.3389/fbioe. 2017.00008/full\#supplementary-material.

Ge, S., Usack, J. G., Spirito, C. M., and Angenent, L. T. (2015). Long-term n-caproic acid production from yeast-fermentation beer in an anaerobic bioreactor with continuous product extraction. Environ. Sci. Technol. 49, 8012-8021. doi:10.1021/acs.est.5b00238

Grootscholten, T. I. M., Steinbusch, K. J. J., Hamelers, H. V. M., and Buisman, C. J. N. (2013). Chain elongation of acetate and ethanol in an upflow anaerobic filter for high rate MCFA production. Bioresour. Technol. 135, 440-445. doi:10.1016/j.biortech.2012.10.165

Guindon, S., Dufayard, J.-F., Lefort, V., Anisimova, M., Hordijk, W., and Gascuel, O. (2010). New algorithms and methods to estimate maximum-likelihood phylogenies: assessing the performance of PhyML 3.0. Syst. Biol. 59, 307-321.780. doi:10.1093/sysbio/syq010

Jeon, B. S., Kim, B. C., Um, Y., and Sang, B. I. (2010). Production of hexanoic acid from D-galactitol by a newly isolated Clostridium sp. BS-1. Appl. Microbiol. Biotechnol. 88, 1161-1167. doi:10.1007/s00253-010-2827-5

Kim, B. C., Seung Jeon, B., Kim, S., Kim, H., Um, Y., and Sang, B. I. (2015). Caproiciproducens galactitolivorans gen. nov., sp. nov., a bacterium capable of producing caproic acid from galactitol, isolated from a wastewater treatment plant. Int. J. Syst. Evol. Microbiol. 65, 4902-4908. doi:10.1099/ijsem.0.000665

Kucek, L. A., Nguyen, M., and Angenent, L. T. (2016). Conversion of L-lactate into n-caproate by a continuously fed reactor microbiome. Water Res. 93, 163-171. doi:10.1016/j.watres.2016.02.018

Lindemann, S. R., Bernstein, H. C., Song, H. S., Fredrickson, J. K., Fields, M. W., Shou, W., et al. (2016). Engineering microbial consortia for controllable outputs. ISME J. 10, 2077-2084. doi:10.1038/ismej.2016.26

Lopetuso, L. R., Scaldaferri, F., Petito, V., and Gasbarrini, A. (2013). Commensal clostridia: leading players in the maintenance of gut homeostasis. Gut Pathog. 5, 23. doi:10.1186/1757-4749-5-23

López-Garzón, C. S., and Straathof, A. J. (2014). Recovery of carboxylic acids produced by fermentation. Biotech. Adv. 32, 873-904. doi:10.1016/ j.biotechadv.2014.04.002

Meerburg, F. A., Vlaeminck, S. E., Ruome, H., Seuntjens, D., Pieper, D. H., Jauregui, R., et al. (2016). High-rate activated sludge communities have a distinctly different structure compared to low-rate sludge communities, and are less sensitive towards environmental and operational variables. Water Res. 100, 137-145. doi:10.1016/j.watres.2016.04.076

Oksanen, J., Blanchet, F. G., Kindt, R., Legendre, P., Minchin, P. R., O’Hara, R. B., et al. (2015). vegan: Community Ecology Package. R Package Version 23-0. Available from: https://cran.r-project.org, https://github.com/vegandevs/ vegan, https://cran.r-project.org/web/packages/vegan/vegan.pdf

Quatravaux, S., Remize, F., Bryckaert, E., Colavizza, D., and Guzzo, J. (2006). Examination of Lactobacillus plantarum lactate metabolism side effects in relation to the modulation of aeration parameters. J. Appl. Microbiol. 101, 903-912. doi:10.1111/j.1365-2672.2006.02955.x

Royce, L. A., Liu, P., Stebbins, M. J., Hanson, B. C., and Jarboe, L. R. (2013). The damaging effects of short chain fatty acids on Escherichia coli membranes. Appl. Microbiol. Biotechnol. 97, 8317-8327. doi:10.1007/s00253-013-5113-5 
Seerdorf, H., Fricke, W. F., Veith, B., Brüggemann, H., Liesegang, H., Strittmatter, A., et al. (2007). The genome of Clostridium kluyveri, a strict anaerobe with unique metabolic features. Proc. Natl. Acad. Sci. U.S.A. 105, 2128-2133. doi:10.1073/pnas.0711093105

Sluiter, A., Hames, B., Ruiz, R., Scarlata, C., Sluiter, J., and Templeton, D. (2006). Determination of Sugars, Byproducts, and Degradation Products in Liquid Fraction Process Samples: Laboratory Analytical Procedure. Golden: National Renewable Energy Laboratory; U.S. Department of Energy; NREL/TP-51042623.

Spirito, C. M., Richter, H., Rabaey, K., Stams, A. J. M., and Angenent, L. T. (2014). Chain elongation in anaerobic reactor microbiomes to recover resources from waste. Curr. Opin. Biotechnol. 27, 115-122. doi:10.1016/j.copbio.2014. 01.003

Steinbusch, K. J. J., Hamelers, H. V. M., Plugge, C. M., and Buisman, C. J. N. (2011). Biological formation of caproate and caprylate from acetate: fuel and chemical production from low grade biomass. Energ. Environ. Sci. 4, 216-224. doi:10.1039/C0EE00282H

Van Eerten-Jansen, M. C. A. A., Ter Heijne, A., Grootscholten, T. I. M., Steinbusch, K. J. J., Sleutels, T. H. J. A., Hamelers, H. V. M., et al. (2013). Bioelectrochemical poduction of caproate and caprylate from acetate by mixed cultures. ACS Sustain. Chem. Eng. 1, 513-518. doi:10.1021/sc300168z

Wang, Y., Naumann, U., Wright, S. T., and Warton, D. I. (2012). mvabund - an R package for model-based analysis of multivariate abundance data. Methods Ecol. Evol. 3, 471-474. doi:10.1111/j.2041-210X.2012.00190.x

Weimer, P. J., and Moen, G. N. (2013). Quantitative analysis of growth and volatile fatty acid production by the anaerobic ruminal bacterium Megasphaera elsdenii T81. Appl. Microbiol. Biotechnol. 97, 4075-4081. doi:10.1007/s00253012-4645-4

Xu, J., Guzman, J. J., Andersen, S. J., Rabaey, K., and Angenent, L. T. (2015). In-line and selective phase separation of medium chain carboxylic acids using membrane electrolysis. Chem. Commun. 51, 6847-6850. doi:10.1039/C5CC01897H

Yang, J., Hou, X., Mir, P. S., and McAllister, T. A. (2010). Anti-Escherichia coli O157: H7 activity of free fatty acids under varying pH. Can. J. Microbiol. 56, 263-267. doi:10.1139/w09-127

Zhou, J., Deng, Y., Zhang, P., Xue, K., Liang, Y., Van Nostrand, J. D., et al. (2013) Stochasticity, succession, and environmental perturbations in a fluidic ecosystem. Proc. Natl. Acad. Sci. U.S.A. 111, E836-E845. doi:10.1073/pnas.1324044111

Zhu, X., Tao, Y., Liang, C., Li, X., Wei, N., Zhang, W., et al. (2015). The synthesis of n-caproate from lactate: a new efficient process for medium-chain carboxylates production. Sci. Rep. 5, 14360. doi:10.1038/srep14360

Conflict of Interest Statement: The authors declare that the research was conducted in the absence of any commercial or financial relationships that could be construed as a potential conflict of interest.

Copyright (C) 2017 Andersen, De Groof, Khor, Roume, Props, Coma and Rabaey. This is an open-access article distributed under the terms of the Creative Commons Attribution License (CC BY). The use, distribution or reproduction in other forums is permitted, provided the original author(s) or licensor are credited and that the original publication in this journal is cited, in accordance with accepted academic practice. No use, distribution or reproduction is permitted which does not comply with these terms. 